\title{
Cambios y Evolución de las Pesquerías en al Atlántico Centro-Oriental ${ }^{1}$
}

\author{
C. BAS \\ Instituto de Investigaciones Pesqueras \\ Barcelona, Espana
}

BAS, C. 1973. Cambios y evolución de las pesquerías en el Atlántico centro-oriental. J. Fish. Res. Board Can. 30: 2301-2307.

Este trabajo se ocupa de la pesca en aguas marinas frente a las costas del Africa occidental, entre el estrecho de Gibraltar y Mauritania, zona que se caracteriza por la relativa estrechez de su plataforma continental, la presencia de la corriente de las Canarias y la intensidad de los afloramientos, que da lugar a una producción primaria elevadísima y una notable abundancia de fauna bentónica.

El autor estudia las principales pesquerías (de sardina, machuelo y alacha, merluza, espáridos, cefalópodos y crustáceos demersales) y analiza su evolución, la composición por especies, los caladeros y estaciones de pesca, las características biológicas de las poblaciones explotadas y los efectos de la pesca.

Se analizan también los criterios que han de tenerse en cuenta a la hora de planificar la inspección y regulación de estas pesquerías.

This paper deals with the marine fisheries off the West African coast, between the Straits of Gibraltar and Mauritania, an area which is characterized by its relatively narrow continental shelf, the presence of the Canary Current, and intensive upwelling resulting in very high primary production and rich bottom fauna.

The main fisheries (for sardines, sardinellas, hakes, sparids, cephalopods, and demersal crustaceans) are analyzed with regard to their evolution, species composition, fishing areas and seasons, the biological characteristics of the exploited stocks, and the effect of fishing on them.

The criteria to be considered when planning the control and regulation of these fisheries are also analysed.

Le présente document traite des pêcheries maritimes opérant au large des côtes de l'Afrique de l'Ouest, entre le détroit de Gibraltar et la Mauritanie. Cetee zone est caractérisée par la relative étroitesse du plateau continental, par la présence du courant des Canaries et par l'intensité de l'upwelling, facteurs qui expliquent l'importance de la production primaire et la richesse de la faune benthique.

L'auteur analyse les principales pêcheries (sardines, sardinelles, merlus, sparidés, céphalopodes et crustacés démersaux) sous divers aspects: évolution, composition par espèces, zaones et époques de pêche, caractéristiques biologiques des stocks exploités et incidences de la pêche sur ces stocks.

Sont également examinés les critères à appliquer dans la planification du contrôle et de la réglementation de ces pêcheries.

LA costa y la plataforma africana en la vertiente atlántica puede considerarse dividida en tres secciones, cada una de las cuales presenta características particulares. En primer lugar se señala la zona comprendida entre el Estrecho de Gibraltar y la región de Cabo Verde; la segunda corresponde a la zona situada desde Angola hasta el Cabo de Bueno Esperanza y finalmente, la zona central está situada en el Golfo de Guinea. Las dos primeras zonas mencionadas se caracterizan por estar situadas en zonas de elevadisima productividad primaria mientras la tercera, por su misma estabilidad, se encuentra en situación mucho menos privilegiada. Aquí se tratará ùnicamente de la primera de las áreas men-

${ }^{1}$ Preparado para la "Conferencia Técnica sobre Ordenacion y Desarrollos Pesqueros," de la Organizacion de las Naciones Unidas para la Agricultura y la Alimentación, celebrada en Vancouver, Colombia Británica, Canadá, del 13 al 23 de febrero de 1973. cionadas, y más concretamente, el área comprendida entre el estrecho de Gibraltar y Mauritania. La plataforma en general no se caracteriza por su gran amplitud, que es màxima a la altura de la península de Río de Oro, donde alcanza unas 75 millas de amplitud entre la costa y la isobata de los $100 \mathrm{~m}$. A esta profundidad normalmente se inicia el talud continental. Tanto en la costa de Marruecos como en la de Mauritania la plataforma es estrecha y muy abrupta en la parte situada al sur de Cabo Blanco. Existen en esta zona dos circunstancias de gran importancia para el conocimiento de la estructura pesquera y de sus posibilidades: la primera es la existencia de la corriente de Canarias y la segunda, los grandes afloramientos que tienen lugar en la zona de Ifni y de Cabo Blanco. La potencia de estos afloramientos es tan considerable que pueden ser considerados como entre los más potentes del mundo dando lugar a elevadas concentraciones de nutrientes en superficie como se desprende de las expediciones oceanográficas llevadas a cabo más recientemente.

Esta grande y duradera aportación de nutrientes a la

Impreso en Canadá (J2945) 
superficie determina el constante enriquecimiento y abundancia de plancton que sostiene una densa población de peces pelágicos pertenecientes a distintas especies cuya explotación es de importancia muy considerable.

Con independencia de estos fenómenos ligados a la dinámica terrestre, en la zona comentada existen importantes áreas de enriquecimiento debidas a la influencia de los vientos cuya componente del NE es la más constante y de la corriente de Canarias, especialmente al rozar ésta con algunos de los salientes litorales. Estas zonas de afloramiento determinan la existencia, entre los grandes afloramientos citados, de una especie de continuidad que se manifiesta especialmente por la riqueza de los fondos, ricos en diversas especies de espáridos, merluzas y cefalópodos, entre los grupos más importantes.

\section{Tipos de pesquerías}

Se trata, en primer lugar, de separar cuidadosamente los tipos de pesca existentes en esta zona; debemos señalar que la zona del gran afloramiento norteño se caracteriza por la existencia de enormes concentraciones de sardina, circunstancia que permite a Marruecos situarse en primera línea en la explotación pesquera mundial. Por el contrario en la zona de Cabo Blanco ha desaparecido la sardina y en su lugar aparece la alacha de características predadores y ecológicas parecidas, especie que se sostiene gracias a la enorme producción marina de esta zona.

\section{Pesquerias pelagicas}

La sardina marroquí se extiende, en general, por toda la costa desde el norte de Villa Cisneros hasta Gibraltar, pero su máxima concentración se encuentra al sur de Marruecos a la altura del paralelo 32. Las circunstancias de alta producción marina y la mayor amplitud de la plataforma en esta zona, permiten la existencia muy firme de un gran stock de esta especie. La explotación está centrada especialmente en Safí y Agadir que son los principales puertos de pesca. Los paises explotadores son principalmente Marruecos y en segundo lugar, pescadores del sur de la península ibérica. En realidad las pesquerías de peces pelágicos, predominantemente sardineras, no se encuentran aisladas sino que colindan con la pesca de extensos cardúmenes de anchoa, ubicados especialmente en la parte norte de la costa marroquí y de alacha que se acerca en bastante cantidad al sur de Villa Cisneros. Las especiales características biológicas de la sardina hacen que no pase hacia el sur más allá del paralelo $25^{\circ} \mathrm{N}$ a causa de la temperatura demasiado elevada de las aguas marinas. Por lo que atañe a la relación existente entre la sardina y la anchoa es importante señalar la posibilidad de sustituciones en los cardùmenes de estas especies, fenómenó observado en otras áreas con las mismas especies y también para otras especies diferentes aunque tengan caracteristicas semejantes. Sin embargo en el momento presente la sardina parece acantonada a la zona de mayor producción marina, mientras la anchoa, menos abundante, se sitúa más al norte que aquélla.

Las características biológicas de la sardina marroquí estudiadas, (Furnestin, Andreu y otros) muestran la existencia de diferencias que afectan tanto al número de vérte- bras $( \pm 51)$ como al de branquispinas $( \pm 80)$ así como a las características del crecimiento, de tal forma que dicha sardina debe ser considerada como una raza localizada en dicha área con caracteristicas estrechamente relacionadas con el ambiente que le es propio, las cuales sin duda es preciso tener en cuenta cuando se programa el control de la explotación de dicha población de peces.

Las pesquerías de alacha o sardinela se situan en la zona de Cabo Blanco lugar donde se encuentran las mayores concentraciones de estos animales. En realidad no existe una población constituida por una sola especie sino que conviven varias de ellas, entre las que hay que destacar Sardinella aurita, Sardinella eba y Sardinella maderensis, ésta última es la menos abundante de las tres. Alcanzan mayor tamaño que la sardina, pero al igual que aquélla, se alimentan de plancton, y debido a la enorme importancia de la producción de esta zona la densidad de estos peces es muy alta. La manera de comportarse de estas especies está menos conocida que la de la sardina por lo que serian de desear intensos estudios sobre las mismas. Se supone que verifican migraciones de norte a sur pero con independencia de los periodos de agregación y dispersión tan frecuentes en las especies de este grupo y de gran importancia para una adecuada explotación de estos bancos de peces. Las sardinelas pasan por períodos de vida en aguas más profundas en las que parece entran a constituir parte importante de la llamada capa difusora profunda. En tal situación son dificilmente capturables, al menos por medio de los habituales artes de cerco. La enorme producción biológica propia de la zona de Cabo Blanco, es la que mantiene la densísima masa de estas especies en estas aguas. Es interesante conocer con más detalle que el actual, los ritmos y oscilaciones en la zona de mayor producción para correlacionarlos con las variaciones en la producción y movimientos de las alachas.

La explotación de estas especies destinadas en el momento actual en gran parte a la conversión en subproductos, se realiza principalmente mediante grandes buques-factoria que destacan flotillas que se dedican a la pesca de los cardúmenes de las mismas. Se utiliza normalmente el arte de cerco, pero algunas flotillas utilizan también artes de arrastre pelágicos con excelentes resultados. Otro aspecto que es interesante señalar aquí es la interacción posible y seguramente existente entre las distintas especies de sardinela. Se trata de cuestiones que deben ser rápidamente experimentadas si se quiere llegar a un auténtico control y a un planteamiento adecuado en la explotación de estas especies. Por lo que atañe a los contactos con especies parecidas hay que señalar que las sardinelas se encuentran en toda la costa norte-africana, sin embargo no son frecuentes en concentraciones tan importantes como sucede en el área que estamos comentando. Al contrario que la sardina, que como se ha indicado, no traspasa hacia el sur del paralelo $25^{\circ} \mathrm{N}$ la alacha se extiende más, por lo que la mezcla de ambos géneros de clupeidos sólo se da en la zona norte. En el sur los contactos son con especies que no llegan a formar concentraciones importantes.

Caracteristicas biológicas - Sardinella aurita y $S$. maderensis son abundantes en la zona. La primera se caracteriza por tener de 100 a 120 branquispinas en la 
parte inferior del primer arco branquial. Se conocen una serie de razas locales características de cada zona. Se la encuentra cerca de la costa y en alta mar. El crecimiento es rápido y al año de vida, el nacimiento tiene lugar en verano cuando el aqua tiene temperatura elevada, alcanza $16 \mathrm{~cm}$ y es apta para la reproducción, circunstancia que favorece grandemente la recuperación de la especie. Cuando las aguas se enfrían en la costa se retira a alta mar. Su alimentación está constituida por plancton de bastante tamaño a causa de la amplitud de la criba de las branquispinas. Alcanza hasta $30 \mathrm{~cm}$ de longitud total. La segunda de las especies es de cuerpo más alto y comprimido. El número de branquispinas varía más ampliamente, entre 90 y $166 \mathrm{~cm}$ quizás en relación con la edad y quizás con una subdivision de especies.

\section{Pesquerias Bentonicas}

Las pesquerias bentónicas tienen una importancia capital en el área que estamos estudiando y han experimentado una tremenda evolución como resultado de la acción de la pesca. Las pescas tradicionales en estas costas estuvieron constituidas por las merluzas en el talud y las zonas más profundas de la plataforma continental en todo el litoral señalado; en este sentido se indican actualmente en la parte sur la fosa de Cayar, cercanías de Cabo Tímiris y Cabo Blanco entre las más productivas. Las merluzas citadas en el área pertenecen a dos especies distintas aunque sus comportamientos son parecidos; se trata de Merluccius merluccius, la merluza europea, de carne blanca y la merluza negra o del Senegal $M$. senegalensis. Podría aceptarse como zona de transición entre ambas especies la de Cabo Blanco, si bien la transgresión entre ellas parece ser considerablemente amplia. Los tamaños medios obtenidos actualmente tanto de la una como de la otra especie, son bastante reducidos y se impone un serio esfuerzo para reducir la intensidad de pesca.

Una segunda e importante pesquería la constituyen los espáridos. En realidad la variedad de tales especies es considerable destacando Dentex macrophthalmus, $D$. dentex, D. canariensis, Spondioliosoma cantharus y especialmente Pagellus belloti y P. acarne entre otras de las especies que tienen interés en esta zona. Desde el punto de vista faunístico las especies pertenecientes al grupo de los espáridos constituyen un importante grupo, con gran número de especies representadas en la zona. En las aguas más profundas se encuentra mayor variedad, tamaños mayores, aunque siempre en cantidad ciertamente reducida; ello se debe en gran parte a la intensa pesca a que han sido sometidos. Por el contrario en aguas menos profundas se encuentra menos variedad y tamaños más pequeños, pero, en ocasiones, gran cantidad de ejemplares.

\section{Cefalopódos}

La tercera de las especies importantes en las pescas de fondo son sin duda los cefalópodos. Tales especies han evolucionado en su aspecto pesquero de modo especial en los últimos doce años y parece que su abundancia corre pareja con la disminución de los espáridos. Se conocen varias áreas en las que los cefalópodos son abundantes; destaca como la más importante la situada entre los paralelos $23^{\circ} \mathrm{N}$ y $25^{\circ} \mathrm{N}$, en fondos más bien cer- canos a la costa y siempre muy poco profundos. Se explotan otras áreas dispersas; una al norte de Cabo Blanco y en la actualidad parece que se pone en explotación otra importante zona situada en las cercanías de Dakar.

En realidad la explotación fundamental de los cefalópodos se basa en tres especies: los pulpos (Octopus vulgaris), la sepia (Sepia officinalis var. hierredda) y el calamar fundamentalmente constituido por Loligo vulgaris. A estas especies hay que añadir cierta cantidad de Loligo forbesi especialmente en aguas alejadas de la costa y diferentes especies del género Sepia también propias de aguas más profundas; éstas últimas se caracterizan por su menor tamaño y también por la menor calidad de su carne; tienen escasa importancia comercial.

De las experiencias llevadas a cabo por diferentes autores se deduce que la zona de puesta del pulpo, especialmente por lo que se refiere a la zona norte (23$\left.25^{\circ} \mathrm{N}\right)$ se sitúa en la parte más superior y siempre cerca de la costa, aprovechando posibles refugios naturales. En general hay que señalar que lejos de la costa, a cierta profundidad, entre 60 y $80 \mathrm{~m}$, se encuentran cierta cantidad de pulpos de gran talla pero en número ciertamente escaso. Las sepias se encuentran prácticamente en la misma área que los pulpos; muestran una acusada disminucion en las capturas, que se manfiesta en la casi desaparición en dichas capturas de la segunda y tercera generación. El calamar también ha disminuido mucho, pero sus costumbres semipelágicas que le permiten separarse del fondo durante largas temporadas del año, le salvaguarda de una pesca demasiado intensa sólo efectiva cuando se acerca al fondo (entrada del calamar). Su área de dispersión es más amplia y está más en consonancia con los movimientos de que es capaz, por lo que parece superior a la de las otras especies. Esta misma circunstancia da como resultado una mayor variabilidad en la composición de las capturas anuales, de tal forma que es difícil separar la evolución debida a la pesca de la evolución propia, debida a su mismo dinamismo.

\section{OTRAS ESPECIES}

Las pesquerías demersales en esta importante área pesquera están complementadas por la existencia de otras especies; entre ellas se destacan las landostas Palinurus regius, Palinurus mauritanicus y $P$. vulgaris. los peneidos Aristeus antennatus, Aristeomorpha foliacea, Plesiopenaeus edwarsianus y Penaeus duorarum en la parte norte y las corvinas, Corvina $s P$., entre las de mayor interés. La corvina se encuentra formando pequeños grupos cerca de la costa y es capturada especialmente cuando verifica movimientos para entrar en las bahías resguardadas, como la de Villa Cisneros y especialmente la del Galgo. Las pesquerias de esta especie han decrecido considerablemente en los tiempos actuales. Las langostas pertenecen a diferentes especies y se encuentran aún con cierta abundancia en las cercanías de Cabo Blanco, en su parte norte. Son intensamente pescadas utilizando las nasas, y son remitidas directamente a los mercados europeos via aérea. Las pesquerías de peneidos son de varias clases y en general se trata de especies que viven en el mismo talud continental. En la costa marroquí se encuentran diferentes especies Aristeus antennatus y Aristeomorpha foliacea. Por el contrario en la parte sur parece ser más abundante Plesiopenaeus edwarsianus. La producción de estas especies 
no puede competir con las pesquerías de camarón de aguas más someras, pero, sin embargo, admiten una mayor capacidad de regulación.

\section{Explotación}

Seguramente lo más interesante en estas diferentes pesquerías demersales lo constituye el diferente comportamiento de estos animales frente a las características del medio, frente a su explotación y el tipo de crecimiento que las caracteriza. En general, tanto las merluzas como los espáridos, aunque principalmente éstos últimos, se caracterizan por un crecimiento relativamente lento y una capacidad de recuperación escasa; esto significa que frente a una pesca intensa se adaptan difícilmente a tal circunstancia. Por otra parte son muy vulnerables debido a la posibilidad de capturarlos en cualquier época del año, e incluso en la edad juvenil. En estas circunstancias una pesquería corre peligro muy serio de su extinción, al menos desde el punto de vista económico.

Por el contrario existen otras pesquerías como las que aprovechan los crustáceos profundos que son capaces de un gran poder de renovación y por su especial y eficaz metabolismo son capaces de mantener un crecimiento intenso y una elevada capacidad de reproducción, de tal forma que, en especial en el caso de Aristeus antennatus son capaces de multiplicarse cuando tan solo tienen un año de vida. Por otra parte los tamaños más solicitados por los intereses de la pesca no resultan perjudiciales para el equilibrio biológico de la población, objeto de la explotación. Los stocks juveniles no reclutados de estas especies se encuentran completamente salvaguardados de la acción de la pesca industrial por sus especiales condiciones ecológicas. En tales casos si bien es cierto que el stock explotado puede mermarse considerablemente, no puede ser rebajado más allá de las condiciones mínimas que representan su crecimiento y el reclutamiento anual.

Cuando se trata de los cefalópodos y en particular del pulpo, sin duda la especie de mayor interés entre las varias explotadas, la experiencia indica que es capaz de un intenso crecimiento, que le permite alcanzar tallas considerables en un tiempo mínimo. Este aspecto se nota muy directamente cuando se trazan las curvas de distribución de tallas, que al contrario de lo que sucede en el caso del calamar y la sepia, se distribuyen formando una única curva de campana, característica de la existencia de una única generación o grupo. Esta circunstancia, junto con su elevado poder reproductor, implica, lo mismo que en otros stocks parecidos, la existencia de un gran poder de regulación y al mismo tiempo la posibilidad de grandes oscilaciones en la abundancia de los stocks muy directamente influenciados por cada generación anual. En tal circunstancia tan sólo grandes series de datos estadísticos que permitan eliminar oscilaciones debidas a circunstancias ajenas a la pesca, servirán para tener un criterio adecuado de la marcha de la pesquería. En estos casos, tanto en el anteriormente citado de los crustáceos de fondo como en el de los pulpos, ocurre algo parecido a las especies de régimen directamente planctófago, como la sardina y las alachas. En todos ellos es más interesante el control de la actividad desde el punto de vista de la economía de las embarcaciones que desde el punto de la acción directa sobre los cardúmenes ya que éstos pueden reducirse de tal forma que el rendimiento por unidad de acción de pesca resulte reducido y sin rentabilidad.

Entre los espáridos y los cefalópodos se ha establecido no obstante una curiosa competición favorecida por la misma acción pesquera. Las zonas comprendidas entre la costa y la isobata de unos $100 \mathrm{~m}$ y en especial en la costa del Sahara fueron tradicionalmente ricas en pesca de espáridos de diferentes especies; la explotación intensiva de estas especies cuyas características son, como se ha indicado, precisamente las opuestas a las señaladas para el caso de los cefalópodos y las gambas de profundidad, es decir, crecimiento no muy intenso y exposición casi total a los artes de pesca, ha dado como consecuencia la continua explotación y disminución de los stocks de los animales. Correlativamente, los celfaópodos fueron aumentando y en especial la pesca del pulpo el cual, como hemos indicado, está afectado de la mayor intensidad de crecimiento; las otras especies no han progresado de la misma forma. Por una parte hay que tener en cuenta que los pulpos constituyen uno de los alimentos más preciados por parte de los espáridos y en segundo lugar que la disminución de la competencia espacial es también de gran interés en el desarrollo de una población de peces, especialmente en una zona donde el potencial de creación de alimentos idóneo - moluscos, pequeños crustáceos, etc - es extraordinario.

Desde el año 1961 acuciados además por el alto interés económico de la nueva pesquería debido a los altos precios alcanzados por los cefalópodos que hasta aquel momento no tenían apenas interés en el mercado mundial (quizás en esto haya jugado un importante papel la entrada de pescadores japoneses con la iniciación de nuevos mercados y utilización de los productos) se intensifica la prospección y se organiza la explotación de estos animales. A partir de esta fecha la explotación se incrementa para alcanzar valores considerables en 1971 en el que un solo barco-factoría dotado de una flotilla de operaciones de unas 50 unidades de arrastreros de tipo medio, elaboraba diariamente un centenar de toneladas de la cuales la mayor parte deben ser consideradas como pulpos. Hay que tener presente que paralelamente a estas actividades están las de otras flotas de las cuales solamente la japonesa y en esta misma zona-banco sahriano-captura la misma cantidad que los pesqueros españoles, representando entre ambos la mayor potencia de explotación en la zona para estas especies.

Cabría pensar si al igual que sucede en otras explotaciones pesqueras la variación en la abundancia o aparente sustitución de unas especies por otras fuera debida a la existencia de fenómenos naturales; ejemplos de este tipo son conocidos de la literatura mundial. Sin embargo, las experiencias realizadas en las campañas oceanográficas más recientemente desarrolladas en la zona y en especial las "Sahara I" y "Atlor I" demuestran que en la misma zona donde se pescan abundantemente los cefalópodos no han dejado de existir considerables cantidades de espáridos entre los que abunda especialmente Pagellus acarne como una de las especies de mayor importancia. Lo que sucede es que el tamaño de los ejemplares es sumamente reducido especialmente en las zonas poco profundas, resultado, sin duda, de la intensa explotación a que fueron sometidos y actualmente, a la competencia 
a que están expuestos por parte de los cefalópodos, en la actualidad ocupantes preferentes del área en cuestión. En tal caso queda muy patente la importancia que tiene el adecuado control de la actividad pesquera que es capaz de desencadenar un cambio tan importante como el indicado, que si bien en el caso concreto citado no ha sido perjudicial pues a un tipo de explotación le ha suplantado otro de gran interés económico, sin embargo lo más probable sería su sustitución por especies de gran poder de crecimiento y adaptación pero carentes de interés para su utilización por parte de la industria de la pesca.

El examen de la totalidad del área demuestra que los espáridos siguen estando ampliamente distribuidos ocupándola totalmente tal como era tradicional, de tal forma que en la costa se encuentran los ejemplares jóvenes y, más alejados en aguas más profundas, un menor número de ejemplares viejos y de gran talla. Algo parecido ocurre con el pulpo que se encuentra igualmente diseminado. Ahora bien, la pesca intensa en la zona típicamente objeto de la explotación de los cefalópodos, impide el adecuado desarrollo de los stocks encargados de nutrir los bancos de espáridos de alta mar y agota prácticamente a los cefalópodos los cuales, por estar mejor adaptados y tener más rápido crecimiento y así mismo un poder de reproducción más elevado, son capaces de mantenerse en condiciones relativamente óptimas en la zona costera objeto de explotación. En concordancia con lo indicado hay que señalar que Ias mayores explotaciones actuales de espáridos llevadas a cabo por las flotas japonesas especialmente de Pagellus belloti, se efectúan al sur de esta zona, en las cercanías de Senegal, donde las explotaciones de cefalópodos son muy inferiores $y$, en todo caso, según se ha indicado más arriba, relativamente recientes.

Resumiendo, en este caso se trata de la acción de la pesca que favoreciendo un lado de la competencia biológica desplaza el equilibrio sistemáticamente hacia el mismo. Actualmente no se está en condiciones de conocer si un adecuado control sería, en el caso de que ello fuera interesante, capaz de retornar la explotación al primitivo auge de los espáridos.

En zonas especialmente situadas cerca de la costa y en algunas áreas de arrastre de plataforma es abundante la langosta. Como consecuencia de su disponibilidad para la pesca y de su lento crecimiento, las langostas han mostrado oscilaciones muy acusadas y en los momentos actuales las especies explotadas son distintamente consideradas por las diferentes flotas que actúan en la explotación, especialmente en lo que atañe a las cercanías de Cabo Blanco. Las flotas francesas que pescan en la zona muestran una acusada disminución, mientras, por el contrario, las flotillas españolas son, al parecer, más rentables en los momentos actuales; sería interesante conocer el resultado de la comparación de los esfuerzos y métodos empleados por cada una de las distintas flotas.

En realidad, una gran parte de la pesca de la zona comprendida en el área del CECAF se nutre de la pesca de peces semipelágicos del grupo de los pequeños escómbridos y de los carángidos; se trata especialmente de la caballa (Scomber japonicus), el chicharro (Trachurus trachurus) y el jurel (Decapterus ronchus) entre otras, a las que pueden acompañar especies de mayor o menor impor- tancia según las épocas. Sólo hay que tener en cuenta que de la totalidad de la pesca consignada para 1968, ligeramente superior a las $2,000,000$ de toneladus métricas, más de una cuarta parte fue capturada por flotillas cuya pesca más importante se centra en la explotación de estas especies. A ello hay que sumar los esfuerzos de otras flotas también muy interesadas en la captura de estos animales.

Son peces que constituyen grandes bancos errantes dentro de pequeñas áreas y que son capaces de interesantes migraciones verticales especialmente durante ciertos períodos del año. Es frecuente que pasen una parte del otoño, mientras se preparan para la próxima freza, en aguas cercanas al fondo y la primera parte del año en aguas superficiales. En tales circunstancias los artes de arrastre de gran abertura vertical, los de cerco y aun los arrastres pelágicos de superficie son los mảs idóneos para su captura. Su crecimiento y poder de reproducción son altos pero así mismo por su comportamiento se encuentran situados más cerca de los peces pelágicos, y especialmente en lo que se refiere a su capacidad de recuperarse de la presión de la pesca. Sin embargo se sabe de algunas especies, por ejemplo la caballa común (Scomber scombrus), que experimentan enormes fluctuaciones a lo largo de los años, seguramente influenciadas por las características del ambiente. Su régimen de alimentación principalmente predador $y$ carnívoro es importante en el período juvenil; entonces actúan directamente sobre el zooplancton beneficiándose de la alta capacidad de producción. de la zona. En el estado adulto la capacidad para ingerir grandes cantidades de elementos constituyentes de la capa difusora profunda permite a estos voraces animales disponer de gran cantidad de alimento muy rico en energía. Experiencias efectuadas con el chicharro (Trachurus trachurus) demuestran la gran cantidad de eufausiáceos de alto poder energético, que son capturados por los cardúmenes de estos peces. Solamente estas circunstancias explican y mantienen la densidad de estos cardúmenes y la posibilidad de que soporten una tan intensa explotación.

\section{Organización de las pesquerías}

Es importante en toda pesquería la acción inmediata que la pesca tiene sobre la estructura de los cardómenes. En el caso de tratarse de pesquerías de animales de superficie y en especial los emigrantes, es posible que el estado de agregación, imprescindible si se quiere lograr éxito en la explotación, dependa, en gran parte, de la acción ambiental: importancia de los agentes físicos y químicos, concentraciones de plancton, etc; pero en el caso de los peces de fondo se demuestra de gran interés para la determinación del estado mínimo de agregación la acción de la propia pesca. Experiencias efectuadas en los bancos de merluza sudafricana y en más pequeñas explotaciones en los fondos de pesca mediterráneos demuestran la importancia de este aspecto. En realidad la explotación de los grandes bancos por un elevado número de unidades pesqueras se caracteriza por la constante rotación en la ubicación de las flotillas de pesca. Las pequeñas áreas donde se detectan ciertas concentraciones de peces, en este caso de cefalópodos, son intensamente explotadas por la mayor concentración de barcos; el resultado es un 
agotamiento rápido de la pequeña agregación y la explotación pasa un período breve, generalmente de uno o dos dias, en busca de nuevas agregaciones, que en cuanto son detectadas concentran rápidamente las flotillas que acaban por agotar el nuevo acúmulo. Siguiendo cuidadosamente el movimiento de estas flotillas durante largos períodos de tiempo, se observa que se van repitiendo los lugares comunes, permaneciendo durante los períodos de explotación en cada punto concreto un lapso de tiempo que varía alrededor de los 5-6 días, en general dependiendo de la concentración real momentánea y también de la intensidad del esfuerzo de pesca que se ejecuta. La disminución en cada situación no es por tanto efecto de una disminución real de la población explotada sino simplemente de la disminución de la agregación existente, por efecto de la misma acción dispersante de la pesca. Este es un aspecto al que se ha prestado escaso interés y consideramos que tiene importancia máxima en el control y en la explotación real de una pesquería. En realidad se precisan técnicas más exactas que las empleadas actualmente en el examen de la pesquerías para poder determinar este parámetro $\mathrm{y}$ aplicarlo a la obtención del máximo rendimiento y mínimo deterioro.

Las pesquerías capaces de mantener un alto nivel de explotación, deben sin duda estar dotadas de una alta capacidad de renovación la cual viene condicionada por las caracteristicas de su biología: alta tasa de crecimiento y elevado poder de reproducción; por otra parte son condiciones idóneas aquéllas que permiten que los stocks juveniles estén automáticamente protegidos de la acción de la pesca. En el caso de las gambas de profundidad, de los pulpos, etc., y también de las pesquerías pelágicas o semipelágicas, se encuentran casi todas estas circunstancias menos las que se refieren a la protección de los cardúmenes juveniles, los cuales, solamente en el caso de las gambas, se encuentran habitualmente protegidos por el estado en que viven las propias larvas.

En estos casos el conocimiento de estos parámetros es condición indispensable si se quiere progresar en el adecuado planteamiento de la explotación de estos recursos. Cuando la explotación es excesiva los stocks se reducen pero sólo hasta tal punto que agota cada año la propia generación. En tales circunstancias, siendo imposible explotar la generación no reclutada por estar fuera del alcance de la pesca, ésta se mantiene a un nivel excesivamente bajo pero estacionario, con ligeras oscilaciones, fruto tan sólo de las influencias ambientales o de los ciclos de producción propios de la especie. El rendimiento sigue disminuyendo por unidad de captura al aumentar el número de éstas sin que de ello se siga que el stock disminuya de forma real. La competencia entre barcos se hace cada ve $z$ más aguda y en tal caso una disminución de la luz de las mallas no representa ninguna ventaja para los pescadores pues se supone que se agota la total generación mientras que un aumento de la malla, en lo que significa de disminución del esfuerzo, repercute mucho en la captura total por barco, medida difícilmente practicable en una situación como la descrita. Estas situaciones se dan de hecho en numerosos casos y en la costa africana centrooccidental se da en los cefalópodos. En tal caso la medida ciertamente interesante es la supresión de un cierto número de unidades de esfuerzo de pesca, pues ni tan solo el establecimiento de cuotas soluciona la cuestión, ya que en tal caso se consigue que la competencia sea la misma pero en un tiempo menor.

Una pesquería adecuadamente organizada en lo que se refiere a una zona concreta como la que nos ocupa debeestar constituida por unas capturas especialmente importantes de peces pelágicos y semipelágicos mediante los cuales se obtiene gran cantidad de peces, destinados a asegurar la masa de la producción con precios relativamente bajos, con lo que no sólo se aseguran los ingresos suficientes sino que además se suministran factores proteínicos suficientes a una gran masa de la población consumidora. Al margen de esta situación, una parte seguramente podría ser industrializada y conservas, seleccionando lo mejor en cada caso, y el producto menos fino podrá ser destinado a la producción de subproductos de tanta importancia en la alimentación animal y para ciertos menesteres industriales. Tal planteamiento evita el que grandes cantidades de peces sean destinados al subproducto en detrimento del consumo humano. Una segunda faceta lo constituyen los peces que reúnen las condiciones anteriormente mencionadas con capacidad de protección y elevada tasa de renovación. En tales casos estas especies son solamente interesantes cuando tienen tasas económicas muy elevadas y cuando sus oscilaciones económicas se mantienen al margen del poder adquisitivo de la población consumidora; se trata especialmente de crustáceos peneidos y cefalópodos. Resta tan sólo considerar aquellas especies que en general constituyen la parte fundamental en las pescas de arraste: por ejemplo los gádidos y los espáridos entre otras y en especial en la zona que nos ocupa. Estas especies precisamente porque su tasa de crecimiento y reproducción no es en general muy alta y por su enorme exposición a la pesca durante largos períodos, entre los que se incluyen, por su habitat, las formas más juveniles, precisan de un control que afecta a la defensa del stock. La ausencia de los controles produce un doble efecto: por una parte la progresiva esquilmación del stock y por otra, la disminución constante de los ingresos por barco. Estos stocks, una vez. deteriorados, son de lenta recuperación y las medidas habitualmente empleadas - tamaño de las mallas, disminución del esfuerzo, etc. - producen resultados satisfactorios, pero en muchas ocasiones se tropieza con dificultades económicas inmediatas que hacen difícil su aplicación.

Anonimo. Exploración oceanográfica del Africa occidental. Campañas del "Malaspina" en 1947 y 1948 en aguas del Sahara, desde Cabo Juby a Punta Durnford. Registro de Operaciones. Bol. Inst. Espan. Oceanogr. 23: $28 \mathrm{p}$.

1950. Exploración oceanográfica del Africa occidental. Campaña del "Malaspina" en enero de 1950 en aguas del Sahara desde Punta Durnford a Cabo Barbas. Bol. Inst. Espan. Oceanogr. 38: 12 p.

1972. Hidrografía de la región de afloramiento del noroeste de Africa. Datos básicos de la Campaña "Sahara II" del Cornide de Saavedra. Res. Exp. Cient. B /O "Cornide" 1: 1-21.

BAs, C. 1971. Campaña Oceanográfica Sahara I. 11 p. (Informe mimeografiado) 
Bas, C, E. Morales, y J. Ma. San Feliu. 1971. Pesquerías de cefalópodos en el banco sahariano. Publ. Tec. Junta Estud. Pesca 9: 129-153.

Cervigón, F. 1960. Peces recogidos en el curso de las campañas realizadas a bordo del "Costa Canaria" desde Cabo Bojador a Guinea Portuguesa (Africa Occidental) y consideraciones sobre su distribución. Invest. Pesq. 17: 33-107.

Garcia Cabrera, C. 1963. El banco pesquero Sahariano. Rev. Estud. Geogr. C.S.I.C.

1966. Investigaciones del Instituto Español de Oceanografía sobre los escómbridos en las aguas ecuatoriales africanas. Publ. Tec. Junta Estud. Pesca 5: 85-143.

1967. Estudio biológico-pesquero de la langosta verde (Palinurus regius Cap.), del litoral sahariano. Publ. Tec. Junta Estud. Pesca 6: 101-139.

1968. Biología y pesca del pulpo (Octopus vulgaris) y choco (Sepia officinalis hierredda) en aguas del Sahara Espanol. Publ. Tec. Junta Estud. Pesca 7: 141-198.

1969. Pulpos y calamares en aguas del Sahara Español. Publ. Tec. Junta Estud. Pesca 8: 75-104.

1970. La pesca en Canarias y Banco Sahariano. Consejo Interprovincial de Canarias. $174 \mathrm{p}$.

Lozano Cabo, F. 1942a. Estudio de la fauna ictiológica de los bancos de Cabo Blanco y de Arguín como tránsito entre la paleártica y la tropical.

1942b. Datos para el conocimiento de la repartición geográfica del Parapritipoma mediterraneum así como de su abundancia en la costa del NW de Africa. Asociación Española para el Progreso de las Ciencias, Oporto.
1948. Relación de una Campaña de pesca de arrastre en pareja en la costa del Sahara Español y noticia sobre los otros tipos de pescas alli practicadas. Bol. Inst. Espan. Oceanogr. 9: 33 p.

Lozano ReY, L. 1934. Las pesquerías del Sahara Español. Publ. Dirección General de Marruecos y colonias, Madrid.

Massutu, M. 1959a. Estudio del crecimiento relativo de Parapenaeus longirrostris, Lucas, del Golfo de Cádiz, Marruecos y Africa occidental francesa. Invest. Pesq. 14: 33-53.

1959b. La gamba (Parapenaeus longirrostris, Lucas) Primeras observaciones en los caladeros del Golfo de Cádiz y Africa occidental. Invest. Pesq. 15: 51-80.

1964. Resultados de la Campaña de prospección pesquera del "Uji-Maru" en aguas del Golfo de Guinea. Publ. Tec. Junta Estud. Pesca 3: 49-131.

1965. La campaña del "Walther Herwig" en aguas del Africa occidental. Publ. Tec. Junta Estud. Pesca 4: 77-205.

1967. Los períodos de pesca en la plataforma continental de Marruecos, Sahara Español, Mauritania, Senegal, R. de Guinea, Ensenada de Biafra y Archipiélago de Cabo Verde. Trab. Inst. Espan. Oceanogr. 34: $100 \mathrm{p}$.

Navarro, F. DE P. 1941. La pesca en la costade Africa desde el Cabo Bojador hasta el banco de Arguin. Ind. Pesq. No. 341:42 p.

Navarro, F. DE P. ET AL. 1943. La pesca de arrastre en los fondos del Cabo Blanco y del Banco de Arguín (Africa sahariana). Trab. Inst. Espan. Oceanogr. 18: $225 \mathrm{p}$. 